J. Indian Chem. Soc.,

Vol. 82, September 2005, pp. 833-837

\title{
Synthesis and biological activity of some new pyrazole derivatives
}

\author{
S. M. Mohamed*, M. Khalile, A. A. Ismail and M. S. Kadh \\ Chemistry Department, Faculty of Science (For Girls), Al-Azhar University, Nasr City, Egypt \\ Manuscript received 21 April 2004, revised 7 January 2005, accepted 20 June 2005
}

\begin{abstract}
1-Substituted-3-(2,4-dimethoxyphenyl)-5-(3,4,5-trimethoxyphenyl)-4,5-dihydropyrazoles were prepared via condensation of 3,4,5-(trimethoxy)benzal-2,4-(dimethoxy)acetophenone with hydrazine derivatives in ethanol and/or condensation with hydrazine hydrate in acidic medium. The product with ethyl iodide, phenyl isothiocyanate, benzoyl chloride and nitrous acid yielded the corresponding l-substituted-3-(2,4-dimethoxyphenyl)-5-(3,4,5-trimethoxyphenyl)-4,5-dihydropyrazoles respectively. The antimicrobial activity of some new compounds has been screened.
\end{abstract}

In the course of recent investigations ${ }^{1,2}$ involving $2,4-$ dimethoxyacetophenone and 3,4,5-trimethoxybenzaldehyde, it was found that 3,4,5-trimethoxybenzal-2,4(dimethoxy)acetophenone (1) is converted into 1,3,5trisubstituted pyrazoles ( 2 and 3 ) by the action of hydrazine derivatives ${ }^{3,4}$ in ethanol or hydrazine hydrate in different acids under reflux. It has been reported that pyrazoles have antimicrobial activities and recently they have respiratory and cardiovascular activities ${ }^{5,6}$.

The aim of this work is to synthesis of some new pyrazoles which might have biological activities ${ }^{7}$.

\section{Results and discussion}

3,4,5-(Trimethoxy)benzal-2,4-(dimethoxy)acetophenone (1) was prepared via condensation of 2,4dimethoxyacetophenone with 3,4,5-trimethoxybenzaldehyde in the presence of base. The ethanolic solution ${ }^{8,9}$ of 1 reacts with hydrazines, namely hydrazine hydrate, phenylhydrazine and/or $p$-tolylsulfonyl hydrazine to afford the corresponding 1,3,5-trisubstituted-4,5dihydropyrazoles (2a-c).

Treatment of acidic solution of $\mathbf{1}$ (formic acid, acetic acid and propionic acid) with hydrazine hydrate under reflux gave the corresponding 1,3,5-trisubstituted-4,5dihydropyrazoles (3a-c, Scheme 1).

Alkylation of $1 \mathrm{H}$-3,5-disubstituted-4,5-dihydropyrazole (2a) with ethyl iodide in presence of anhydrous potassium carbonate in dry acetone, gave the corresponding 1ethyl-3,5-disubstituted-4,5-dihydropyrazole (4). The structure of 4 was also established via reduction ${ }^{10}$ of 1 -acetyl3,5-disubstituted-4,5-dihydropyrazole (3b) with lithium aluminum hydride in ether. Treatment of $2 \mathrm{a}$ with phenyl isothiocyanate in ether produced 1-(phenylamino)thiocarbonyl-4,5-disubstituted-3,5-dihydropyrazole (5, Scheme 1).

Compound 2a was allowed to react with benzoyl chloride and $p$-toluene-sulphonyl chloride in pyridine to give the corresponding 1-benzoyl-3,5-disubstituted-4,5dihydropyrazole (6) and 1-p-tolylsulphonyl-3,5-disubstituted-4,5-dihydropyrazole (2c), respectively.

Bromination of $1 H$-3-(2,4-dimethoxyphenyl)-5-(3,4,5trimethoxyphenyl)-4,5-dihydropyrazole (2a) by bromine in chloroform gave the corresponding $1 \mathrm{H}$-4-bromo-3-(2,4dimethoxyphenyl)-5-(3,4,5-triethoxyphenyl pyrazole (6, Scheme 1).

Treatment of $1 \mathrm{H}$-3,5-disubstituted-4,5-dihydropyrazole (2a) with nitrous acid afforded the corresponding 1-nitroso3-(2,4-dimethoxyphenyl)-5-(3,4,5-trimethoxyphenyl)-4,5dihydropyrazole (8, Scheme 1).

\section{Biological activity :}

Using the agar plate diffusion technique ${ }^{11}$ with concentrations of 75, 125, 175 and $250 \mathrm{ppm}$, all the newly synthesized compounds were tested for antimicrobial effect against Staphylococcus aureus, Bacillus cereus, Serratia marcesens, Proteus merabitis and Aspergillus fumgytus. After $24 \mathrm{~h}$ the growth in the broth was evaluated visually according to its zone of inhibition. The screening results (given in Table 1) indicated that the compounds $2 c$ and 7 exhibited marked activity against different types of bacteria, while the compounds $2 \mathbf{a}$ and 3b exhibited moderate activity against different types of bacteria. The results indicated that all newly synthesized compounds 2a, 2c, 3b and 7 exhibited moderate activity against Aspergillus fumgytus with concentration $250 \mathrm{ppm}$. 
J. Indian Chem. Soc., Vol. 82, September 2005

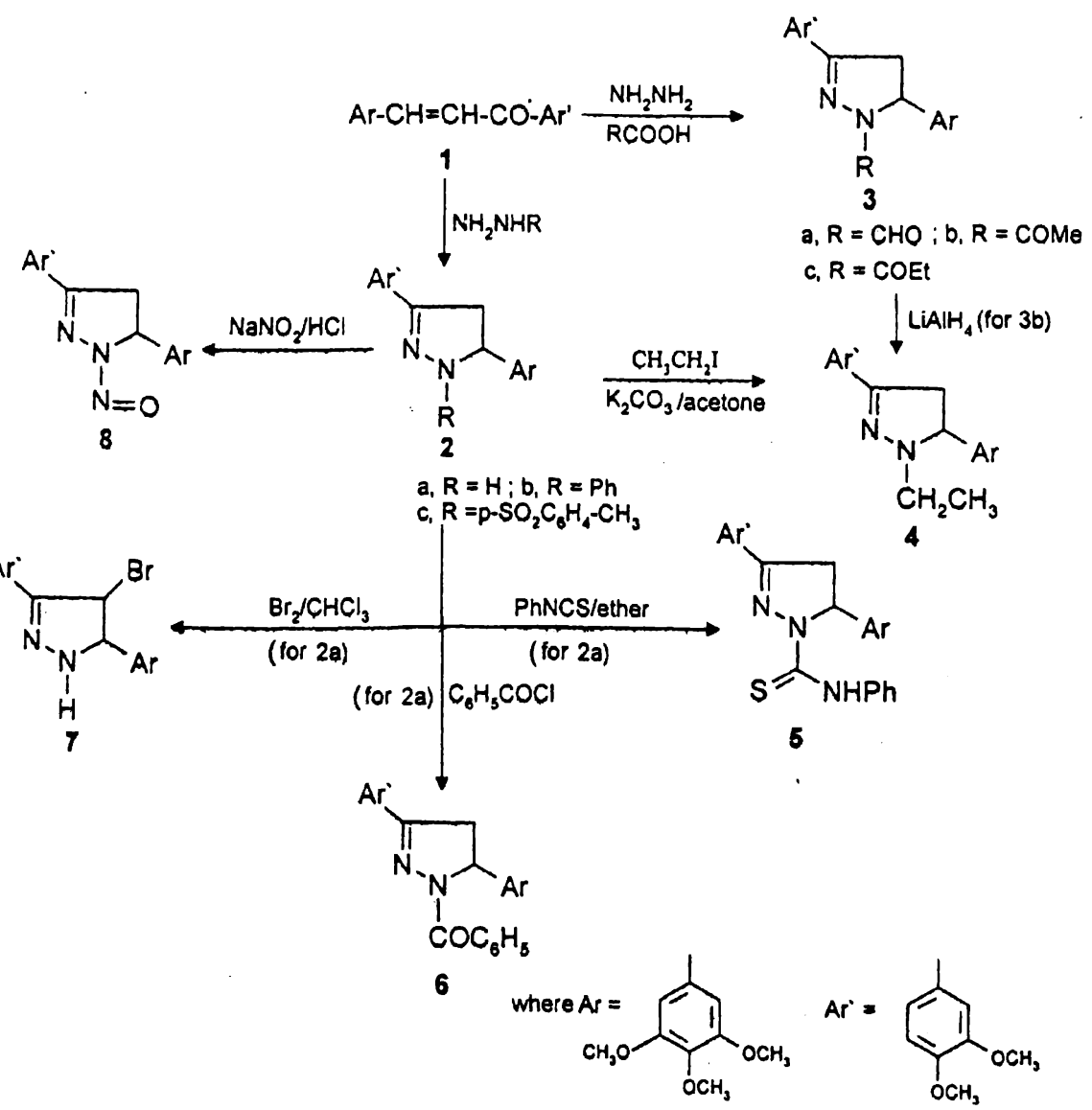

Scheme 1

\section{Experimental}

IR spectra were recorded as $\mathrm{KBr}$ pellet on a Pye Unicom SP 2000 spectrophotometer and PMR spectra on a Varian EM-360-60 MHz NMR spectrometer using TMS internal standard. Mass spectra were obtained on a GCMSQP 100 Ex mass spectrometer. Microanalysis were conducted using an elemental analyzer 1106, in Faculty of
Science, Cairo University. Melting points were determined on a Richter Hot stage and uncorrected.

1-Substituted-3-(2,3-dimethoxyphenyl)-5-(3,4,5trimethoxyphenyl)-4,5-dihydropyrazoles (2a-c) :

A mixture of $1(0.01 \mathrm{~mol})$, hydrazine hydrate, phenylhydrazine and/or $p$-toluenesulphonyl hydrazine $(0.01 \mathrm{~mol})$ in ethanol $(30 \mathrm{ml})$ was heated for $5 \mathrm{~h}$. After

Table 1. Antimicrobial activity of some synthesized compounds at different concentrations (ppm)

\begin{tabular}{|c|c|c|c|c|c|c|c|c|c|c|c|c|c|c|c|c|c|c|c|c|}
\hline \multirow{2}{*}{$\begin{array}{l}\text { Compd.I } \\
\text { Concn. }\end{array}$} & \multicolumn{4}{|c|}{$\begin{array}{c}\text { S. aureus } \\
\text { (ATCC-6538-P) }\end{array}$} & \multicolumn{4}{|c|}{$\begin{array}{c}\text { B. cereus } \\
\text { (NRRL-B-569) }\end{array}$} & \multicolumn{4}{|c|}{$\begin{array}{l}\text { S. marcesens } \\
\text { (IMRV-70) }\end{array}$} & \multicolumn{4}{|c|}{$\begin{array}{l}\text { P. merabitis } \\
\text { (NTC-289) }\end{array}$} & \multicolumn{4}{|c|}{$\begin{array}{c}\text { A. fumgytus } \\
\text { (PP-29) }\end{array}$} \\
\hline & 75 & 125 & 175 & 250 & 75 & 125 & 175 & 250 & 75 & 125 & 175 & 250 & 75 & 125 & 175 & 250 & 75 & 125 & 175 & 250 \\
\hline $2 a$ & + & + & ++ & +++ & + & + & ++ & ++ & + & ++ & ++ & +++ & + & ++ & ++ & ++ & - & - & ++ & ++ \\
\hline $2 c$ & + & ++ & +++ & +++ & + & + & +++ & +++ & + & ++ & ++ & +++ & + & + & ++ & +++ & - & - & + & ++ \\
\hline $3 \mathbf{b}$ & + & + & + & ++ & + & + & ++ & ++ & + & + & ++ & ++ & + & + & ++ & ++ & - & + & + & ++ \\
\hline 7 & + & ++ & +++ & +++ & + & ++ & +++ & +++ & + & ++ & ++ & +++ & + & + & ++ & +++ & - & - & + & ++ \\
\hline
\end{tabular}


Mohamed et al. : Synthesis and biological activity of some new pyrazole derivatives

cooling, the product was filtered off, dried and purified by recrystallization with ethanol to give 2 .

1H-3-(2,4-Dimethoxyphenyl)-5-(3,4,5-trimethoxyphenyl)-4,5-dihydropyrazole (2a) was obtained as colourless crystals, m.p. $95^{\circ} \mathrm{C}$, yield $62 \%$. $v_{\max }(\mathrm{KBr})$ $3221(\mathrm{NH}), 1620(\mathrm{C}=\mathrm{N}), 1035(\mathrm{C}-\mathrm{O}) \mathrm{cm}^{-1} ; \delta_{\mathrm{H}}\left(\mathrm{CDCl}_{3}\right)$ 3.70-3.72 (2H, d, $\left.\mathrm{CH}_{2} \mathrm{CH}\right), 3.76\left(3 \mathrm{H}, \mathrm{s}, \mathrm{OCH}_{3}\right), 3.78$ $\left(3 \mathrm{H}, \mathrm{s}, \mathrm{OCH}_{3}\right), 3.80\left(3 \mathrm{H}, \mathrm{s}, \mathrm{OCH}_{3}\right), 3.91\left(3 \mathrm{H}, \mathrm{s}, \mathrm{OCH}_{3}\right)$, $3.95\left(3 \mathrm{H}, \mathrm{s}, \mathrm{OCH}_{3}\right), 5.48\left(1 \mathrm{H}, \mathrm{t}, \mathrm{CHCH}_{2}\right), 6.91-7.63$ (5H, m, Ar-H), $10.31(1 \mathrm{H}, \mathrm{s}, \mathrm{NH}) \mathrm{ppm} . \mathrm{m} / \mathrm{z}(\%) 372$ $\left(\mathrm{M}^{+}, 46.30\right), 371\left(\mathrm{M}^{+}-1,76.35\right), 194$ (38.30) (Found : C, 64.63; H, 6.39; N, 7.60. $\mathrm{C}_{20} \mathrm{H}_{24} \mathrm{~N}_{2} \mathrm{O}_{5}$ Calcd. : C, $64.51 ; \mathrm{H}, 6.45 ; \mathrm{N}, 7.53 \%)$.

1-Phenyl-3-(2,4-dimethoxyphenyl)-5-(3,4,5-trimethoxy)-4,5-dihydropyrazole (2b) was obtained as pale yellow crystals, yield $81 \%$, m.p. $71^{\circ} \mathrm{C} . v_{\max }(\mathrm{KBr}) 1615$ $(\mathrm{C}=\mathrm{N}), 1605,1585(\mathrm{C}=\mathrm{C}), 1037,1012(\mathrm{C}-\mathrm{O}) \mathrm{cm}^{-1} ; \delta_{\mathrm{H}}$ $\left(\mathrm{CDCl}_{3}\right) 3.71-3.73\left(2 \mathrm{H}, \mathrm{dd}, \mathrm{CH}_{2} \mathrm{CH}\right), 3.75(3 \mathrm{H}, \mathrm{s}$, $\left.\mathrm{OCH}_{3}\right), 3.77\left(3 \mathrm{H}, \mathrm{s}, \mathrm{OCH}_{3}\right), 3.80\left(3 \mathrm{H}, \mathrm{s}, \mathrm{OCH}_{3}\right), 3.90$ $\left(3 \mathrm{H}, \mathrm{s}, \mathrm{OCH}_{3}\right), 3.94\left(3 \mathrm{H}, \mathrm{s}, \mathrm{OCH}_{3}\right), 5.48(1 \mathrm{H}, \mathrm{t}$, $\left.\mathrm{CHCH}_{2}\right), 6.91-7.81(9 \mathrm{H}, \mathrm{m}, \mathrm{Ar}-\mathrm{H}) \mathrm{ppm} . \mathrm{m} / \mathrm{z}(\%) 449$ $\left(\mathrm{M}^{+}+1,1.35\right), 448\left(\mathrm{M}^{+}, 23.20\right), 371$ (53.03), 194 (21.21), 193 (17.81), 77 (25.30) (Found : C, 69.80; H, 6.30; N, 6.40. $\mathrm{C}_{26} \mathrm{H}_{28} \mathrm{~N}_{2} \mathrm{O}_{5}$ Calcd. : C, 69.64; H, 6.25; $\mathrm{N}, 6.25 \%$ ).

1-p-Tolylsulphonyl-3-(2,4-dimethoxyphenyl)-5-(3,4,5trimethoxyphenyl)-4,5-dihydropyrazole (2c) was obtained as pale yellow crystals, yield $68 \%$, m.p. $180^{\circ} \mathrm{C} . v_{\max }$ $(\mathrm{KBr}) 1617(\mathrm{C}=\mathrm{N}), 1603,1590(\mathrm{C}=\mathrm{C}), 1120,1033$, $1010(\mathrm{C}-\mathrm{O}) \mathrm{cm}^{-1} ; \delta_{\mathrm{H}}\left(\mathrm{CDCl}_{3}\right) 2.30\left(3 \mathrm{H}, \mathrm{s}, \mathrm{CH}_{3}\right), 3.70-$ $3.72\left(2 \mathrm{H}, \mathrm{dd}, \mathrm{CH}_{2} \mathrm{CH}\right), 3.76\left(3 \mathrm{H}, \mathrm{s}, \mathrm{OCH}_{3}\right), 3.78(3 \mathrm{H}$, $\left.\mathrm{s}, \mathrm{OCH}_{3}\right), 3.81\left(3 \mathrm{H}, \mathrm{s}, \mathrm{OCH}_{3}\right), 3.91\left(3 \mathrm{H}, \mathrm{s}, \mathrm{OCH}_{3}\right)$, $3.95\left(3 \mathrm{H}, \mathrm{s}, \mathrm{OCH}_{3}\right), 5.49\left(1 \mathrm{H}, \mathrm{t}, \mathrm{CHCH}_{2}\right), 6.91-7.80$ (8H, m, Ar-H) ppm. m/z (\%) $527\left(\mathrm{M}^{+}, 11.31\right), 526$ $\left(\mathrm{M}^{+}-1,23.51\right), 372$ (23.51), 371 (100), 205 (46.33), 163 (17.31), 156 (23.05), 91 (21.32) (Found : C, 61.63; $\mathrm{H}, 5.63 ; \mathrm{N}, 5.50 ; \mathrm{S}, 5.89 . \mathrm{C}_{27} \mathrm{H}_{30} \mathrm{~N}_{2} \mathrm{O}_{7}$ S Calcd. : C, $61.60 ; \mathrm{H}, 5.70 ; \mathrm{N}, 5.32 ; \mathrm{S}, 6.08 \%)$.

1-Substituted-3-(2,4-dimethoxyphenyl)-5-(3,4,5-trimethoxyphenyl)-4,5-dihydropyrazole (3a-c) :

A mixture of 1 ( $0.01 \mathrm{~mol})$ and hydrazine hydrate $(0.01$ mol) in acid (such as formic acid, acetic acid and propionic acid) ( $30 \mathrm{ml}$ ) was heated for $5 \mathrm{~h}$ after cooling, the formed solid was filtered off, dried and purified by recrystallization from ethanol to give 3 .

1-Formyl-3-(2,4-dimethoxyphenyl)-5-(3,4,5-trimethoxyphenyl)-4,5-dihydropyrazole (3a) was obtained as pale yellow crystals, yield $81 \%$, m.p. $140^{\circ} \mathrm{C}$. $v_{\max }(\mathrm{KBr})$ $1640(\mathrm{C}=\mathrm{O}), 1613(\mathrm{C}=\mathrm{N}), 1604,1591(\mathrm{C}=\mathrm{C}), 1210$, $1030,1015(\mathrm{C}-\mathrm{O}) \mathrm{cm}^{-1} ; \delta_{\mathrm{H}}\left(\mathrm{CDCl}_{3}\right) 3.71-3.73(2 \mathrm{H}, \mathrm{dd}$, $\left.\mathrm{CH}_{2} \mathrm{CH}\right), 3.76\left(3 \mathrm{H}, \mathrm{s}, \mathrm{OCH}_{3}\right), 3.78\left(3 \mathrm{H}, \mathrm{s}, \mathrm{OCH}_{3}\right), 3.80$ $\left(3 \mathrm{H}, \mathrm{s}, \mathrm{OCH}_{3}\right), 3.90\left(3 \mathrm{H}, \mathrm{s}, \mathrm{OCH}_{3}\right), 3.94\left(3 \mathrm{H}, \mathrm{s}, \mathrm{OCH}_{3}\right)$, $5.49\left(1 \mathrm{H}, \mathrm{t}, \mathrm{CHCH}_{2}\right), 6.90-7.65(5 \mathrm{H}, \mathrm{m}, \mathrm{Ar}-\mathrm{H}), 9.42$ $(1 \mathrm{H}, \mathrm{s}, \mathrm{CHO}) \mathrm{ppm} . \mathrm{m} / \mathrm{z}(\%) 401\left(\mathrm{M}^{+}+1,1.30\right), 400$ $\left(\mathrm{M}^{+}, 21.56\right), 399\left(\mathrm{M}^{+}-1,33.61\right), 372(63.52), 194$ (38.91), 193 (22.05), 163 (19.23) (Found : C, 62.89; H, 6.10; N, 6.83. $\mathrm{C}_{21} \mathrm{H}_{24} \mathrm{~N}_{2} \mathrm{O}_{6}$ Calcd. : C, 63.00; H, 6.00; $\mathrm{N}, 7.00 \%)$.

1-Acetyl-3-(2,4-dimethoxyphenyl)-5-(3,4,5-trimethoxyphenyl)-4,5-dihydropyrazole (3b) was obtained as pale yellow crystals, yield $62 \%$; m.p. $130^{\circ} \mathrm{C} . v_{\max }(\mathrm{KBr})$ $1698(C=O), 1613(C=N), 1604,1595(C=C), 1210$, 1038, $1015(\mathrm{C}-\mathrm{O}) \mathrm{cm}^{-1} ; \delta_{\mathrm{H}}\left(\mathrm{CDCl}_{3}\right) 2.41(3 \mathrm{H}, \mathrm{s}$, $\left.\mathrm{COCH}_{3}\right), 3.70-3.72\left(2 \mathrm{H}\right.$, dd, $\left.\mathrm{CH}_{2} \mathrm{CH}\right), 3.74(3 \mathrm{H}, \mathrm{s}$, $\left.\mathrm{OCH}_{3}\right), 3.76^{\circ}\left(3 \mathrm{H}, \mathrm{s}, \mathrm{OCH}_{3}\right), 3.79\left(3 \mathrm{H}, \mathrm{s}, \mathrm{OCH}_{3}\right), 3.91$ $\left(3 \mathrm{H}, \mathrm{s}, \mathrm{OCH}_{3}\right), 3.95\left(3 \mathrm{H}, \mathrm{s}, \mathrm{OCH}_{3}\right), 5.49\left(1 \mathrm{H}, \mathrm{t}, \mathrm{CHCH}_{2}\right)$, 6.90-7.80 (5H, m, Ar-H) ppm. $m / z(\%) 415\left(\mathrm{M}^{+}+1\right.$, 9.31), $414\left(\mathrm{M}^{+}, 13.56\right), 372$ (53.62), 371 (43.61), 163 (18.25) (Found : $\mathrm{C}, 63.50 ; \mathrm{H}, 6.41 ; \mathrm{N}, 6.70 . \mathrm{C}_{22} \mathrm{H}_{26} \mathrm{~N}_{2} \mathrm{O}_{6}$ Calcd. : C, 63.77; H, 6.28; N, 6.76\%).

1-Propionyl-3-(2,4-dimethoxyphenyl)-5-(3,4,5-trimethoxyphenyl)-4,5-dihydropyrazole (3c) as pale yellow crystals, yield $70 \%$, m.p. $65^{\circ} \mathrm{C} . v_{\max }(\mathrm{KBr}) 1695(\mathrm{C}=\mathrm{O})$, $1614(\mathrm{C}=\mathrm{N}), 1603,1559(\mathrm{C}=\mathrm{C}), 1230,1039,1012(\mathrm{C}-$ O) $\mathrm{cm}^{-1} ; \delta_{\mathrm{H}}\left(\mathrm{CDCl}_{3}\right) 1.2\left(3 \mathrm{H}, \mathrm{t}, \mathrm{CH}_{3}\right), 2.53(2 \mathrm{H}, \mathrm{q}$, $\left.\mathrm{COCH}_{2}\right), 3.71-3.73\left(2 \mathrm{H}\right.$, dd, $\left.\mathrm{CH}_{2} \mathrm{CH}\right), 3.75(3 \mathrm{H}, \mathrm{s}$, $\left.\mathrm{OCH}_{3}\right), 3.77\left(3 \mathrm{H}, \mathrm{s}, \mathrm{OCH}_{3}\right), 3.79\left(3 \mathrm{H}, \mathrm{s}, \mathrm{OCH}_{3}\right), 3.90$ $\left(3 \mathrm{H}, \mathrm{s}, \mathrm{OCH}_{3}\right), 3.94\left(3 \mathrm{H}, \mathrm{s}, \mathrm{OCH}_{3}\right), 5.46(1 \mathrm{H}, \mathrm{t}$, $\left.\mathrm{CHCH}_{2}\right), 6.93-7.76(5 \mathrm{H}, \mathrm{m}, \mathrm{Ar}-\mathrm{H}) \mathrm{ppm} . \mathrm{m} / \mathrm{z}(\%) 429$ $\left(\mathrm{M}^{+}+1,3.61\right), 428\left(\mathrm{M}^{+}, 16.35\right), 372(51.31), 371$ (63.25), 164 (20.31), 163 (17.20) (Found : C. 64.31; H, 6.40; N, 6.60. $\mathrm{C}_{23} \mathrm{H}_{28} \mathrm{~N}_{2} \mathrm{O}_{6}$ Calcd. : C, 64.49; H, 6.54; $\mathrm{N}, 6.54 \%)$.

1-Ethyl-3-(2,4-dimethoxyphenyl)-5-(3,4,5-trimethoxyphenyl)-4,5-dihydropyrazole (4) :

A mixture of $3 \mathbf{b}(0.01 \mathrm{~mol})$ and lithium aluminum hydride $(0.01 \mathrm{~mol})$ in dry ether $(30 \mathrm{ml})$ was heated on water bath for $5 \mathrm{~h}$. While hot, the reaction mixture was filtered and excess of solvent was removed by evaporation. The solid obtained was purified by recrystallization from methanol to give 4 as pale yellow crystals, yield $45 \%$, m.p. $300^{\circ} \mathrm{C}$. $v_{\max }(\mathrm{KBr}) 1615(\mathrm{C}=\mathrm{N}), 1605,1598$ $(\mathrm{C}=\mathrm{C}), 1210,1031,1012(\mathrm{C}-\mathrm{O}) \mathrm{cm}^{-1} ; \delta_{\mathrm{H}}\left(\mathrm{DMSO}-d_{6}\right)$ $1.21\left(3 \mathrm{H}, \mathrm{t}, \mathrm{CH}_{3}\right), 3.01\left(2 \mathrm{H}, \mathrm{q}, \mathrm{CH}_{2}\right), 3.70-3.72(2 \mathrm{H}$, 
dd, $\left.\mathrm{CH}_{2} \mathrm{CH}\right), 3.75\left(3 \mathrm{H}, \mathrm{s}, \mathrm{OCH}_{3}\right), 3.77\left(3 \mathrm{H}, \mathrm{s}, \mathrm{OCH}_{3}\right)$, $3.79\left(3 \mathrm{H}, \mathrm{s}, \mathrm{OCH}_{3}\right), 3.90\left(3 \mathrm{H}, \mathrm{s}, \mathrm{OCH}_{3}\right), 3.94(3 \mathrm{H}, \mathrm{s}$, $\left.\mathrm{OCH}_{3}\right), 6.93-7.70(5 \mathrm{H}, \mathrm{m}, \mathrm{Ar}-\mathrm{H}) \mathrm{ppm} . \mathrm{m} / \mathrm{z}(\%) 401$ $\left(\mathrm{M}^{+}+1,9.61\right), 400\left(\mathrm{M}^{+}, 18.32\right), 372(52.56), 371$ (63.62), 194 (19.63), 163 (17.68) (Found : C, 65.81; H, $6.90 ; \mathrm{N}, 7.10 . \mathrm{C}_{22} \mathrm{H}_{28} \mathrm{~N}_{2} \mathrm{O}_{5}$ Calcd. : C, 66.00; H, 7.00; $\mathrm{N}, 7.00 \%)$.

\section{Alkylation of 2a with ethyl iodide, Formation of 4 :}

A mixture of $2 \mathrm{a}(0.01 \mathrm{~mol})$, ethyl iodide $(0.01 \mathrm{~mol})$ and anhydrous potassium carbonate $(0.03 \mathrm{~mol})$ in dry acetone $(30 \mathrm{ml})$ heated fo: $15 \mathrm{~h}$. The reaction mixture was cooled and poured into water. The obtained solid was filtered off, washed with water, dried and purified by recrystallization with methonal to give 4 .

1-(Phenylamino)thiocarbonyl-3-(2,3-dimethoxyphenyl)5-(3,4,5-trimethoxyphenyl)-4,5-dihydropyrazole (5) :

A mixture of $2 \mathrm{a}(0.01 \mathrm{~mol})$ and phenyl isothiocyanate $(0.01 \mathrm{~mol})$ in ether $(30 \mathrm{ml})$ was heated on water bath for $5 \mathrm{~h}$. After evaporation of the solvent the obtained product was purified by recrystallization from ethanol to give 5 as yellow crystals, yield $45 \%$, m.p. $115^{\circ} \mathrm{C}$. $v_{\max }(\mathrm{KBr})$ $3225(\mathrm{NH}), 1620(\mathrm{C}=\mathrm{N}), 1605,1591(\mathrm{C}=\mathrm{C}), 1318$ $(\mathrm{C}=\mathrm{S}), 1121,1035,1013(\mathrm{C}-\mathrm{O}) \mathrm{cm}^{-1} ; \delta_{\mathrm{H}}\left(\mathrm{CDCl}_{3}\right) 3.71-$ $3.72\left(2 \mathrm{H}, \mathrm{dd}, \mathrm{CH}_{2} \mathrm{CH}\right), 3.75\left(3 \mathrm{H}, \mathrm{s}, \mathrm{OCH}_{3}\right), 3.78(3 \mathrm{H}$, $\left.\mathrm{s}, \mathrm{OCH}_{3}\right), 3.81\left(3 \mathrm{H}, \mathrm{s}, \mathrm{OCH}_{3}\right), 3.40\left(3 \mathrm{H}, \mathrm{s}, \mathrm{OCH}_{2}\right)$, $3.95\left(3 \mathrm{H}, \mathrm{s}, \mathrm{OCH}_{3}\right), 5.48\left(1 \mathrm{H}, \mathrm{t}, \mathrm{CHCH}_{2}\right), 6.91-7.73$ $(10 \mathrm{H}, \mathrm{m}, \mathrm{Ar}-\mathrm{H}), 10.32(1 \mathrm{H}, \mathrm{s}, \mathrm{NH}) \mathrm{ppm} . \mathrm{m} / \mathrm{z}(\%) 508$ $\left(\mathrm{M}^{+}+1,8.03\right), 507\left(\mathrm{M}^{+}, 19.35\right), 372(100), 371$ (52.11), 205 (33.90), 163 (32.21), 135 (19.25) (Found : C, 63.80; $\mathrm{H}, 5.70 ; \mathrm{N}, 8.32 ; \mathrm{S}, 6.09 . \mathrm{C}_{27} \mathrm{H}_{29} \mathrm{~N}_{3} \mathrm{O}_{5}$ S Calcd. : C, $63.91 ; \mathrm{H}, 5.72 ; \mathrm{N}, 8.28 ; \mathrm{S}, 6.31 \%)$.

1-Benzoyl-3-(2,4-dimethoxyphenyl)-5-(3,4,5-trimethoxyphenyl)-4,5-dihydropyrazole (6) :

A mixture of $2 \mathrm{a}(0.01 \mathrm{~mol})$ and benzoyl chloride $(0.01$ mol) in pyridine $(15 \mathrm{ml})$ was heated on water bath for $2 \mathrm{~h}$. The reaction mixture was cooled and acidified with $4 N$ hydrochloric acid. The obtained solid was filtered off, washed with water, dried, and purified by recrystallization from ethanol to give 6 as pale yellow crystals, yield $63 \%$, m.p. $100^{\circ} \mathrm{C}$. $v_{\max }(\mathrm{KBr}) 1695(\mathrm{C}=\mathrm{O}), 1619$ $(\mathrm{C}=\mathrm{N}), 1604,1593(\mathrm{C}=\mathrm{C}), 1120,1030,1015(\mathrm{C}-\mathrm{O})$ $\mathrm{cm}^{-1} ; \delta_{\mathrm{H}}\left(\mathrm{CDCl}_{3}\right) 3.70-3.72\left(2 \mathrm{H}, \mathrm{dd}, \mathrm{CH}_{2} \mathrm{CH}\right), 3.75$ $\left(3 \mathrm{H}, \mathrm{s}, \mathrm{OCH}_{3}\right), 3.78\left(3 \mathrm{H}, \mathrm{s}, \mathrm{OCH}_{3}\right), 3.80\left(3 \mathrm{H}, \mathrm{s}, \mathrm{OCH}_{3}\right)$, $3.90\left(3 \mathrm{H}, \mathrm{s}, \mathrm{OCH}_{3}\right), 3.94\left(3 \mathrm{H}, \mathrm{s}, \mathrm{OCH}_{3}\right), 5.49(1 \mathrm{H}, \mathrm{t}$, $\left.\mathrm{CHCH}_{2}\right), 6.93-7.78(10 \mathrm{H}, \mathrm{m}, \mathrm{Ar}-\mathrm{H}) \mathrm{ppm} . \mathrm{m} / \mathrm{z}(\%) 477$ $\left(\mathrm{M}^{+}+1,5.21\right), 476\left(\mathrm{M}^{+}, 52.50\right), 372(12.90), 371$ (62.50), 312 (25.40), 194 (16.60), 105 (100), 77 (39.90)
(Found : C, 68.20; H, 5.68; N, 5.80. $\mathrm{C}_{27} \mathrm{H}_{28} \mathrm{~N}_{2} \mathrm{O}_{6}$ Calcd. : C, $68.07 ; \mathrm{H}, 5.88 ; \mathrm{N}, 5.88 \%)$.

IH-3-(2,4-Dimethoxyphenyl)-4-bromo-5-(3,4,5-trimethoxyphenyl)pyrazole (7) :

A solution of $2 \mathrm{a}(0.01 \mathrm{~mol})$ in chloroform $(20 \mathrm{ml})$ was added dropwise to a solution of bromine $(0.03 \mathrm{~mol})$ in chloroform $(20 \mathrm{ml})$ with stirring for $15 \mathrm{~min}$. After addition the mixture was strirred for further $30 \mathrm{~min}$. The formed solid was collected, washed with water, dried and purified by recrystallization from acetone to give 7 as yellow crystals, yield $83 \%$, m.p. $182^{\circ} \mathrm{C}$. $v_{\max }(\mathrm{KBr})$ $3225(\mathrm{NH}), 1625(\mathrm{C}=\mathrm{N}), 1605,1585(\mathrm{C}=\mathrm{C}), 1250,1038$, $1012(\mathrm{C}-\mathrm{O}) \mathrm{cm}^{-1} ; \delta_{\mathrm{H}}\left(\mathrm{DMSO}-d_{6}\right) 3.75\left(3 \mathrm{H}, \mathrm{s}, \mathrm{OCH}_{3}\right)$, $3.77\left(3 \mathrm{H}, \mathrm{s}, \mathrm{OCH}_{3}\right), 3.81\left(3 \mathrm{H}, \mathrm{s}, \mathrm{OCH}_{3}\right), 3.90(3 \mathrm{H}, \mathrm{s}$, $\left.\mathrm{OCH}_{3}\right), 3.95\left(3 \mathrm{H}, \mathrm{s}, \mathrm{OCH}_{3}\right), 6.95-7.60(5 \mathrm{H}, \mathrm{m}, \mathrm{Ar}-\mathrm{H})$, $10.63(1 \mathrm{H}, \mathrm{s}, \mathrm{NH}) \mathrm{ppm}$ (Found : C, 53.30; H, 4.56; N, 6.10. $\mathrm{C}_{20} \mathrm{H}_{23} \mathrm{BrN}_{2} \mathrm{O}_{5}$ Calcd. : C, 53.22; H, 5.10; N, $6.21 \%)$.

1-Nitroso-3-(2,4-dimethoxyphenyl)-5-(3,4,5-trimethoxyphenyl)-4,5-dihydropyrazole (8) :

A solution of $2 \mathrm{a}(0.01 \mathrm{~mol})$ in concentrated hydrochloric acid $(10 \mathrm{ml})$ was chilled in ice to $-5^{\circ} \mathrm{C}$. A cold solution of the sodium nitrite $(0.02 \mathrm{~mol})$ in water (10 $\mathrm{mL}$ ) was added dropwise with stirring for $30 \mathrm{~min}$. The precipitate was collected, washed with water, dried and purified by recrystallization from acetone to give $\mathbf{8}$ as yellow crystals, yield $35 \%$, m.p. $125^{\circ} \mathrm{C}$. $v_{\max }(\mathrm{KBr}) 1616$ $(\mathrm{C}=\mathrm{N}), 1605,1595(\mathrm{C}=\mathrm{C}), 1490(\mathrm{~N}=\mathrm{O}), 1210,1035$, $1015(\mathrm{C}-\mathrm{O}) \mathrm{cm}^{-1} ; \delta_{\mathrm{H}}\left(\mathrm{CDCl}_{3}\right) 3.71-3.73\left(2 \mathrm{H}, \mathrm{d}, \mathrm{CH}_{2} \mathrm{CH}\right)$, $3.73\left(3 \mathrm{H}, \mathrm{s}, \mathrm{OCH}_{3}\right), 3.78\left(3 \mathrm{H}, \mathrm{s}, \mathrm{OCH}_{3}\right), 3.81(3 \mathrm{H}, \mathrm{s}$, $\left.\mathrm{OCH}_{3}\right), 3.90\left(3 \mathrm{H}, \mathrm{s}, \mathrm{OCH}_{3}\right), 3.94\left(3 \mathrm{H}, \mathrm{s}, \mathrm{OCH}_{3}\right), 5.49$ $\left(1 \mathrm{H}, \mathrm{t}, \mathrm{CHCH}_{2}\right), 6.91-7.70(5 \mathrm{H}, \mathrm{m}, \mathrm{Ar}-\mathrm{H}) \mathrm{ppm} . \mathrm{m} / \mathrm{z}$ (\%) $402\left(\mathrm{M}^{+}+1,13.50\right), 401\left(\mathrm{M}^{+}, 27.10\right), 371$ (56.35), 205 (17.50), 163 (29.10) (Found : C, 59.67; H, 5.74; N, 10.50. $\mathrm{C}_{20} \mathrm{H}_{23} \mathrm{~N}_{3} \mathrm{O}_{6}$ Calcd. : C, 59.85; H, 5.74; N, $10.47 \%)$.

\section{References}

1. ZJ-Jia, Y. Wu, W. Huang, P. Zhang, L. A. Clizbe, E. A. Goldman, U. Sinha, A. Clizbe, E. A. Goldman, U. Sinha, A. E. Arfsten, S. T. Edwards, M. Alphonso, A. Hutchaleelaha, R. M. Scarborough and B. Yzhu, Bioorg. \& Medicinal Chem. Lett., 2004, 14, 1221.

2. L. Deluca, G. Giacomelli, A. Porcheddu, M. Salaris, M. Salaris and M. Taddei, C. R. Chimie, 2003, 6, 607.

3. M. A. Saleh, M. F. Abdel-Megeed, M. A. Abdo and M. S. Abdel-Basset, Molecules, 2003, 8, 363. 
Mohamed et al. : Synthesis and biological activity of some new pyrazole derivatives

4. L. Commeiras, S. C. Woodcock, J. E. Baldwin, R. M. Adlington, A. R. Cowlex and P. D. Wilkinson, Tetrahedron, 2004, 60, 933.

5. A. H. El-Masry, H. H. Fahmy and S. H. A. Abdelwahed, Molecules, 2000, 5, 1429.

6. R. Anikwue, J. W. Huffman et al., J. Pharmacol. Exp. Ther., 2002, 303, 340.

7. M. A. Berghot and E. B. Moawad, European J. Pharm. Sci.,
2003, 20, 173.

8. Algaforova and Mileklina, Doke Akad Nauk, 1966, 171, 125.

9. Grandberg, Kost Zhur Ibshchei, 1960, 30, 1373 (Chem. Abst., 1961, 55, 518).

10. G. H. Sayed and H. Kjsen, J. Prak. Chemie, 1980, 322, 716.

11. J. C. Gould and J. M. Boure, Med. J. Chem., 1952, 178, 59. 